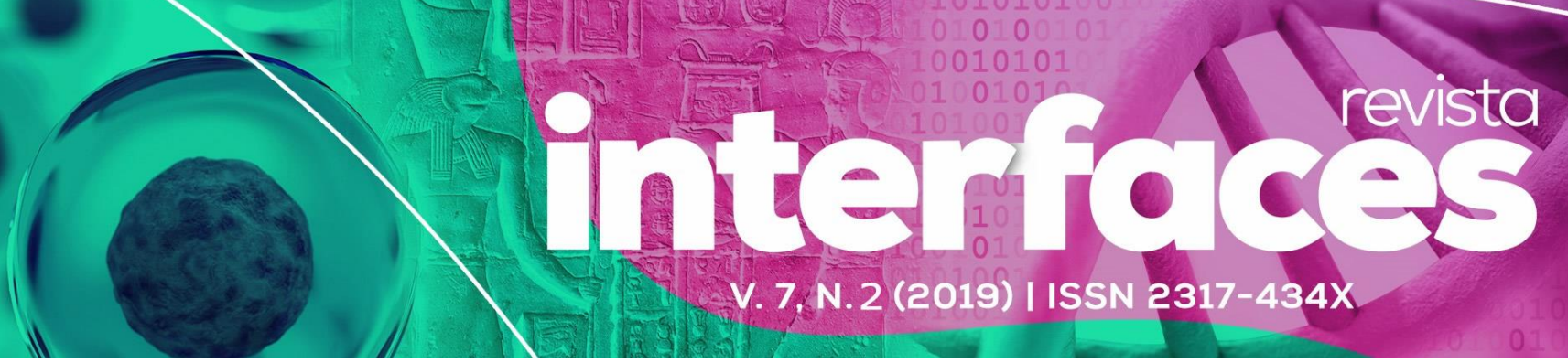

\title{
SAÚDE DO TRABALHADOR: CONDIÇÕES DE SAÚDE EM MULTIPROFISSIONAIS COM ÊNFASE NO EXAME PERIÓDICO E ACIDENTES RELACIONADOS AO TRABALHO
}

\author{
HEALTH OF WORKER: HEALTH CONDITIONS IN MULTIPROFESSIONAL WITH EMPHASIS IN \\ THE PERIODIC EXAM AND WORK-RELATED ACCIDENTS
}

DOI: 10.16891/2317-434X.v7.e2.a2019.pp266-273

Recebido em: 28.02.2019 / Aceito em: 09.08.2019

\begin{abstract}
Thaina Freire Quinteiro Silva, Hercules Pereira Coelho*, Camila Maria do Nascimento, Isabelly Rayane Alves dos Santos, Ana Beatriz Linard de Carvalho, Ana Paula Ribeiro de Castro, Mônica Maria Viana da Silva, Ana Maria Machado Borges
\end{abstract}

Centro Universitário Doutor Leão Sampaio - UNILEÃO Av. Leão Sampaio $\mathrm{km} 3$ - Lagoa Seca Juazeiro do Norte - CE - CEP 63040-005 E-mail: herculesleon_01@yahoo.com

\section{RESUMO}

As discussões acerca da saúde do trabalhador surgiram devido ao grande índice de acidentes de trabalho no Brasil pela exposição a vários riscos neste ambiente, como: riscos ergonômicos, físicos, de acidentes, e outros. O estudo tem como objetivo avaliar as condições de saúde em multiprofissionais com ênfase no exame periódico e acidentes relacionados ao trabalho. Trata-se de uma pesquisa de caráter descritivo, documental, com abordagem quantitativa, realizada em uma clínica de engenharia e medicina do trabalho, localizada no município de Salgueiro, Pernambuco. A amostra foi composta por 14 prontuários de trabalhadores atendidos na clínica. O estudo foi realizado no período de fevereiro a novembro de 2018. Para a coleta de dados foi elaborado um formulário. O projeto foi aprovado pelo Comitê de Ética em Pesquisa do Centro Universitário Dr. Leão Sampaio, com o parecer no 2.913.774. A maioria dos profissionais era do sexo masculino $(85,7 \%)$, com idade entre 25 e 56 anos, exercendo as funções de servente $(28,6 \%)$ e talheiro (14,3\%), estando expostos a riscos físicos $(42,9 \%)$ e de acidente (50,0\%). Todos os trabalhadores realizaram o exame admissional (100\%). Os trabalhadores que adoeceram no período trabalhado registraram as seguintes causas: cirurgia de apendicectomia, fratura óssea (falanges ou clavículas), dorsalgia e fratura do antebraço, no entanto, não configuraram acidentes de trabalho, pois ocorreram em outros ambientes. Portanto, a partir dos dados apresentados, é perceptível a necessidade de acompanhar e orientar rigorosamente cada trabalhador no período trabalhado, com o intuito de reduzir os riscos e os agravos à saúde.

Palavras chave: Saúde do trabalhador; Acidentes de trabalho; Exame periódico.

\section{ABSTRACT}

The discussions about health of workers came about due to the high rate of work accidents in Brazil due to exposure to various risks in this environment, such as: ergonomic, physical, accident, and other risks. The aim of this study is to evaluate health conditions in multiprofessional, with emphasis on periodic examination and work-related accidents. This is a descriptive, documentary, quantitative approach, carried out in a clinic of engineering and occupational medicine, located in the city of Salgueiro, Pernambuco. The sample consisted of 14 medical records of workers attended at the clinic. The study was carried out from February to November, 2018. A form was prepared for data collection. The project was approved by the Ethics Committee in Research of the University Center Dr. Leão Sampaio, with the opinion no 2.913 .774 . The majority of the professionals were male $(85,7 \%)$, aged 25 to 56 years old, working as servant $(28,6 \%)$ and carter $(14,3 \%)$, being exposed to physical risks $(42,9 \%)$ and accident $(50,0 \%)$. All the workers performed the admission examination (100\%). Workers who fell ill during the period worked reported the following causes: appendectomy surgery, bone fracture (phalanges or clavicles), dorsalgia and forearm fracture. However, they did not configure work accidents because they occurred in other environments. Therefore, from the data presented, it is noticeable the need to follow and strictly guide each worker in the period worked, in order to reduce risks and health problems.

Keywords: Health of worker; Accidents at work; Periodic exam. 


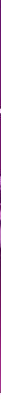

masculino, e que os tipos de riscos mais prevalentes são o físico e o de acidente. Em consideração a NR7, os itens básicos estão sendo parcialmente obedecidos, pois no que tange o exame admissional foi averiguado, por meio dos prontuários, que todos os trabalhadores realizaram o mesmo no período hábil, ao passo que houve discrepância quanto à realização do exame periódico, visto que alguns funcionários foram submetidos a mais exames que $o$ preconizado, e outros a menos.

De acordo com os resultados evidenciados neste estudo, é importante que haja o desenvolvimento de práticas seguras para a prevenção e promoção da saúde destes trabalhadores, tais como: os exames admissional e periódico serem realizados no período trabalhado; orientações quanto às condutas que devem ser tomadas em caso de adoecimento no trabalho; discutir sobre as possíveis soluções para os problemas encontrados (riscos); esclarecer as dúvidas em caso de acidentes de trabalho, e outros.

O estudo apresentou algumas limitações, como por exemplo, a coleta de dados ter sido realizada através dos prontuários e arquivos dos trabalhadores assistidos pela clínica, o que restringe a coleta aos dados que estavam registrados no sistema de informação, não podendo, os mesmos, serem confrontados com os trabalhadores.

O trabalho tem como sugestões a realização de uma pesquisa com os casos de adoecimento no trabalho; analisar a percepção dos trabalhadores doentes, podendo assim dialogar e definir como se sentem por estarem afastados do trabalho; realizar um estudo sobre as principais causas de acidentes no trabalho; e comparar o acidente físico com as práticas de segurança desenvolvidas no trabalho.

\section{REFERÊNCIAS}

BEZERRA, G. Análise dos riscos ergonômicos e das doenças ocupacionais nos canteiros de obras e formas de prevenção [MONOGRAFIA]. Universidade Tecnológica Federal do Paraná, Campo Mourão, 2015. Acesso em: 18 de agosto de 2018. Disponível

em:

<http://repositorio.roca.utfpr.edu.br/jspui/bitstream/1/ 5847/1/CM_COECI_2014_2_03.pdf>

BORDONI, P. H. C.; BORDONI, L. S. contribuição da autópsia médico-legal para a investigação do acidente de trabalho fatal: relato de caso. Rev. Bras Med Trab., n. 4, v. 15, p.372-377. 2017.

BRASIL. Ministério da Saúde. Diretrizes e normas regulamentadoras de pesquisa envolvendo seres humanos, Resolução no 466, de Dezembro de 2012. Brasília: Diário Oficial da União da República Federativa do Brasil, 2012.

BRASIL. Normas Regulamentadoras. NR 7 Programa de Controle Médico de Saúde Ocupacional (107.000-2). Dispõe sobre o Programa de Controle Médico de Saúde Ocupacional - PCMSO. 2018. Acesso em: 02 de novembro de 2018. Disponível em:
http://www.trtsp.jus.br/geral/tribunal2/LEGIS/CLT/N Rs/NR_7.html

GELATI, T. R. et al. Alterações respiratórias em trabalhadores: estudo de prontuários avulsos. Rev. Gaúcha Enferm. n. 4, v. 38, p. 1-9. 2017.

GOMEZ, C. M.; VASCONCELLOS, L. C. F.; MACHADO, J. M. H. Saúde do trabalhador: aspectos históricos, avanços e desafios no Sistema Único de Saúde. Ciência \& Saúde Coletiva, 23(6):1963-1970. 2018.

LEAL, E. N.; LIMA, J. G. M. Riscos ergonômicos em uma empresa de extração de óleos vegetais. Rev. Baiana de Saúde Pública, n. 1, v. 41, p. 98-119. 2017.

MATURINO, M. M.; FERNANDES, R. C. P.; RÊGO, M. A. V. A atuação do SUS na vigilância de ambientes de trabalho: a experiência do Centro Estadual de Referência em Saúde do Trabalhador (CESAT) na Bahia. Rev. Bras. Saúde Ocup. N. 1, v. 43, p. 1-13. 2018.

MENDES, E. A.; TEIXEIRA, L. R.; BONFATTI, R. $\mathrm{J}$. As condições de saúde dos trabalhadores a partir dos 


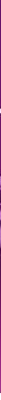

exames periódicos de saúde. Rev. Saúde Debate, n. 112, v. 41, p. 142-154. 2017.

MILANI, D. et al. Obstáculos para retorno ao trabalho: tradução de adaptação cultural do questionário para o contexto brasileiro. Ciência \& Saúde coletiva. n. 5, v. 23, p. 1387-1401. 2018.

PIMENTEL, B. N. et al. Percepção do ruído, saúde auditiva e qualidade de vida de professores de escolas públicas. Audiolcommum Res. n. 1, v. 21, p. 1-7. 2016.

PINA, J. A.; STOTZ, E. N.; JACKSON FILHO, J. M. J. Trabalhador "compatível", fratura exposta no processo de produção da indústria automobilística: intensificação do trabalho e saúde em questão. Cad. Saúde Pública, n. 7, v. 34, p. 1-13. 2018.

SILVA, A. R.; ARAÚJO, T. M. Acidentes de trabalho grave no estado da bahia no período de 2007 a 2012. Rev. Baiana de Saúde Pública, n. s2, v. 40, p. 57-69. 2016.

SILVA, E. S. N.; SANTOS, T. F. V. Análise dos padrões técnicos de programas de controle médico de saúde ocupacional e atestados de saúde ocupacional por meio de auditoria interna. Rev. Bras Med Trab., n.2,v.12 ,p. 50-56. 2014.

SILVA, J. O.; LUZ, M. L. S. Ergonomia e segurança do trabalho em uma empresa prestadora de manutenção hidráulica [MONOGRAFIA]. Departamento de Engenharia de Produção. Universidade Estadual de Maringá - UEM. Campus Sede - Paraná, Brasil. 2018. Acesso em: 25 de outubro de 2018. Disponível em: http://www.dep.uem.br/gdct/index.php/dep_tcc/articl e/view/1587

SILVA, P. S. F.; SANTOS, W. L.; AMARAL, E. Principais dificuldades enfrentadas pelos profissionais do serviço especializado em engenharia da segurança e medicina do trabalho dentro da construção civil. Rev Inic Cient Ext. 1(Esp3):304-12. 2018.
SILVA-JUNIOR, J. S. A saúde do trabalhador que cuida da saúde. Rev. BrasMed Trab., n. 3, v. 15, p. 193. 2017.

SOUTO, C. C. et al. Perfil das vítimas de acidentes de transporte terrestre relacionados ao trabalho em unidades de saúde sentinelas de pernambuco. Epidemiol. Serv. Saúde. n. 2, v. 25, p. 351-361. 2016.

SOUZA, G. F. et al. Fatores de riscos ocupacionais e implicações à saúde do trabalhador em biotérios. Rev. Saúde Debate, n. especial, v. 41, p. 180-199. 2017. 